\title{
Irradiation Effects in Compact Binaries
}

\author{
Martin E. Beer \& Philipp Podsiadlowski \\ Oxford University, Wilkinson Building, Keble Road, Oxford OX1 3RH, \\ $U K$
}

\begin{abstract}
Irradiation of the secondaries in close binary systems affects their appearance and can drastically change their internal structure and hence long-term evolution. In this paper we review the main effects of external irradiation in close binaries with compact primaries, such as the distortion of the shape of the secondary and the conditions for the occurrence of outer critical configurations, and apply these to normal Xray binaries, including systems such as $\mathrm{HZ}$ Her/Her X-1, Vela X-1 and black-hole binaries during outburst (e.g. Nova Sco). Particular emphasis will be given to the role of circulation driven by the external heating and the radiative surface stress. We have developed a three-dimensional fluid dynamics code for modelling these effects. We present initial results of self-consistent calculations for the circulation in irradiated systems and show how the inclusion of these effects is vital to the understanding and interpretation of any system where external irradiation of the secondary is significant.
\end{abstract}

\section{Introduction}

Irradiation of the secondaries in close binary systems can dramatically affect their appearance both spectroscopically and photometrically. X-ray heating of the stellar surface changes the observed light curve. In Nova Sco (Orosz \& Bailyn 1997) it has been observed that the quiescent magnitude is dimmer than the outburst magnitude for all phases. The shape of the light curve itself is altered with a primary minimum that is much deeper than the secondary minimum during outburst. Heating also affects the observed radial-velocity curve. In SS Cyg in outburst, the observed radial-velocity amplitude is $50 \mathrm{~km} \mathrm{~s}^{-1}(30 \%)$ larger than that observed during quiescence (Hessman et al. 1984). Heating also distorts the radial-velocity curve from a pure sinusoid. In Nova Sco (Phillips, Shahbaz, \& Podsiadlowski 1999) the residuals of a fit to the observed absorptionline curve when compared to a standard sinusoidal fit are as large as $20 \mathrm{~km} \mathrm{~s}^{-1}$ $(10 \%)$. In addition, in Vela X-1 there are persistent residuals in the radialvelocity curve $\left(5 \mathrm{kms}^{-1}, 25 \%\right.$, Barziv et al. 2001) whose cause is unknown. These could be a direct observation of circulation currents on the secondary surface.

$\mathrm{X}$-ray heating also affects regions not directly irradiated. In $\mathrm{HZ} \mathrm{Her}$ the stellar-surface brightness distribution appears to be inverted (Wilson 1973a,b), while in Nova Sco (Shahbaz et al. 2000) an indirectly heated region is required 
in order to explain the observed increase of the radial-velocity curve amplitude during outburst (30\%). As the primary mass is proportional to the radialvelocity amplitude cubed, the X-ray heating significantly affects the calculation of the primary mass and hence our interpretation of the evolutionary states of these systems. In particular, in persistent systems where the quiescent radialvelocity amplitude is never observed, the contribution of the X-ray heating is crucial to our understanding of the components and the evolutionary state. For a discussion indicating how the evolutionary interpretation can change for different secondary masses in Nova Sco see Beer \& Podsiadlowski (2002a).

Irradiation can also change the internal structure and hence long-term evolution of close binary systems. In low-mass X-ray binaries (LMXBs) masstransfer rates appear to be about an order of magnitude larger than the standard model predicts (Ruderman et al. 1989). A possible cause of this could be an irradiation-driven wind, alternatively irradiation-driven expansion may be the missing ingredient (see $\S 2$ for a more detailed discussion).

\section{Irradiation effects on the stellar structure}

Apart from heating the outer layers of the star and raising the surface temperature, irradiation affects the stellar structure in several fundamental ways:

- The radiation-pressure force itself, acting on the surface layer of the star, deforms the stellar surface. Phillips \& Podsiadlowski (2002) modelled the surface as an equipotential surface where the effect of external irradiation was taken into account through a modified potential. This treatment was only approximate, since the radiative force is not a conservative force and hence cannot strictly be represented by a potential (see Phillips \& Podsiadlowski 2002 for more detail). Drechsel et al. (1995) have also considered the effect of radiation pressure on stellar surfaces but without considering the shadowing effect of an accretion disc.

- Heating of the surface layers causes temperature gradients along equipotential surfaces. This drives circulation from the heated surface to the cooler regions (see Kippenhahn \& Thomas 1979 and related discussion in $\S 3)$.

- The radiation-pressure force causes a surface stress whose gradient drives horizontal motion perpendicular to the stress and vertical motion in a thin turbulent boundary layer (an Ekman layer), a process known as Ekman pumping. This process is entirely analogous to the wind-driven circulation in oceanic circulation systems (see Chapter 5 of Pedlosky 1987).

- Irradiation changes the surface boundary conditions of the star which can lead to long-term structural changes since it affects the efficiency by which internal energy is radiated away at the surface. This could lead to significant expansion of the secondary (Podsiadlowski 1991). Ritter, Zhang, \& Kolb (2000) and Hameury (1996) have both considered the case of anisotropic irradiation.

All of these effects need to be considered if a global solution is to be found. 


\section{Previous work}

The irradiation-induced circulation has been modelled previously but only in simplified models. Kippenhahn \& Thomas (1979) considered HZ Her as a twolevel model in planar geometry using perturbation theory. They found a circulation pattern whose depth was $20 \%$ of the stellar radius and whose horizontalvelocity component was supersonic. Kurbıyık (1982) following on from Kırbıyık \& Smith (1976) also used perturbation theory but in spherical geometry. They found a circulation pattern which extended on to the unilluminated hemisphere whose velocities were supersonic. Martin \& Davey (1995) used a 2D smooth particle hydrodynamics (SPH) model with the particles free to move between equipotential surfaces of the secondary in the orbital plane of the system. They found a circulation pattern which extended completely over the stellar surface which had a maximum velocity of $31.7 \mathrm{~km} \mathrm{~s}^{-1}$ when the surface temperature was increased by $36,000 \mathrm{~K}$.

In summary a circulation pattern develops in the upper layers of the star which reaches the unilluminated side. When the surface is not deformed the tangential-velocity component of this circulation is supersonic. Phillips \& Podsiadlowski (2002) found that with a modified potential and assuming zero circulation there was a large surface deformation. Comparing this to the previous work we see two extremes. No deformation leads to a supersonic velocity but no circulation leads to a high deformation. In reality the solution will be somewhere between the two and so any model which is successful in modelling circulation must include the effects of circulation and deformation simultaneously.

\section{Our model}

From the previous discussion it is clear that the problem of irradiation-driven circulation requires accurate treatment of the surface geometries and boundary conditions, three dimensions and therefore supercomputing facilities. We have developed a general three-dimensional hydrodynamics code (Beer \& Podsiadlowski 2002b) for modelling this circulation. The code is based on methods developed for ocean circulation and has a surface-normalised grid (so as to accurately model the surface geometry). It includes the effect of the Coriolis force (which has been neglected in all previous models) and models processes which occur on the dynamic timescale. Consequently we assume zero mass loss from the surface as any mass loss over a dynamical timescale will be negligible. Currently it has a polytropic equation of state and so does not solve the thermodynamic equation, although a future extension will. Hence the present models are not fully realistic. This simplified model will still allow, however, a quantitative determination of the three-dimensional circulation systems. The code has been successfully tested on standard problems (see Beer \& Podsiadlowski 2002b for details).

There are effects other than circulation which could contribute to the energy transport in compact binaries. These include convection and differential rotation. Convection will be important in the layer below the layer in which the temperature inversion (due to heating) occurs. It will act mainly in the direction of the effective gravity. It may also be possible to get energy transport by 
large-scale turbulence (i.e. weather). Currently we do not consider the possible effects of differential rotation as we expect these systems to be tidally locked. However, it still could be important if the velocity shear in the outer layer is comparable to the circulation velocity.

The code models processes on the dynamic timescale but one of the aims of the project is to understand the evolution through the expansion of the secondary. This occurs on a thermal timescale and it may appear as if there is a conflict of timescales. The resolution of this is simple, however, due to the large difference in the two timescales. There is no need to model the circulation throughout the length of the thermal timescale expansion but only when the background model changes significantly. Hence, only a few calculations of the circulation pattern need to be made over the thermal timescale in order to find the accurate circulation pattern and stellar model.

To investigate the possible extent of the circulation pattern over the surface we have carried out two-dimensional calculations with our code. As these are two dimensional they do not include the effect of the Coriolis force - but can give an indication of the extent of the circulation currents over the stellar surface when irradiation and distortion are both considered. We assumed typical parameters for a star in a LMXB - one solar mass and two solar radii with a polytropic index of 1.5 (appropriate for convective stars). We assumed that it was spherical and applied an irradiating force to the uppermost layer of the star. We used an X-ray luminosity of $2 \times 10^{31} \mathrm{~W}$, near the Eddington limit of a neutron star, and a separation of the components of 5 solar radii. Figure 1 shows the circulation pattern reached after 118 dynamical time-steps. Comparing this to the result of Martin \& Davey (1995) we see that the extent of the circulation current is the same. This is because there is no Coriolis force in either calculation and so there is nothing to stop the flow once it has started until it meets itself on the unilluminated side of the star. The magnitude of the circulation, however, is subsonic unlike the previous work. This is because the surface is free to adjust and so can deform to compensate. Only a small deformation is necessary in this case to make the circulation subsonic.

\section{Conclusions}

Irradiation dramatically changes the observable properties of close binaries. Understanding irradiation effects is crucial for the reliable determination of system parameters. X-ray irradiation drives circulation currents in the upper layers of the secondaries of compact binaries. Understanding by how much the secondary expands as a result of the external irradiation will allow a better modelling of the mass-transfer rates in systems where irradiation effects are important.

\section{References}

Barziv, O., Kaper, L., Van Kerwijk, M. H., Telting, J. H., \& Van Paradijs, J. 2001, A\&A, 377, 925

Beer, M. E., \& Podsiadlowski, Ph. 2002a, MNRAS, 331, 351

Beer, M. E., \& Podsiadlowski, Ph. 2002b, MNRAS, in press (astro-ph/0109484) 


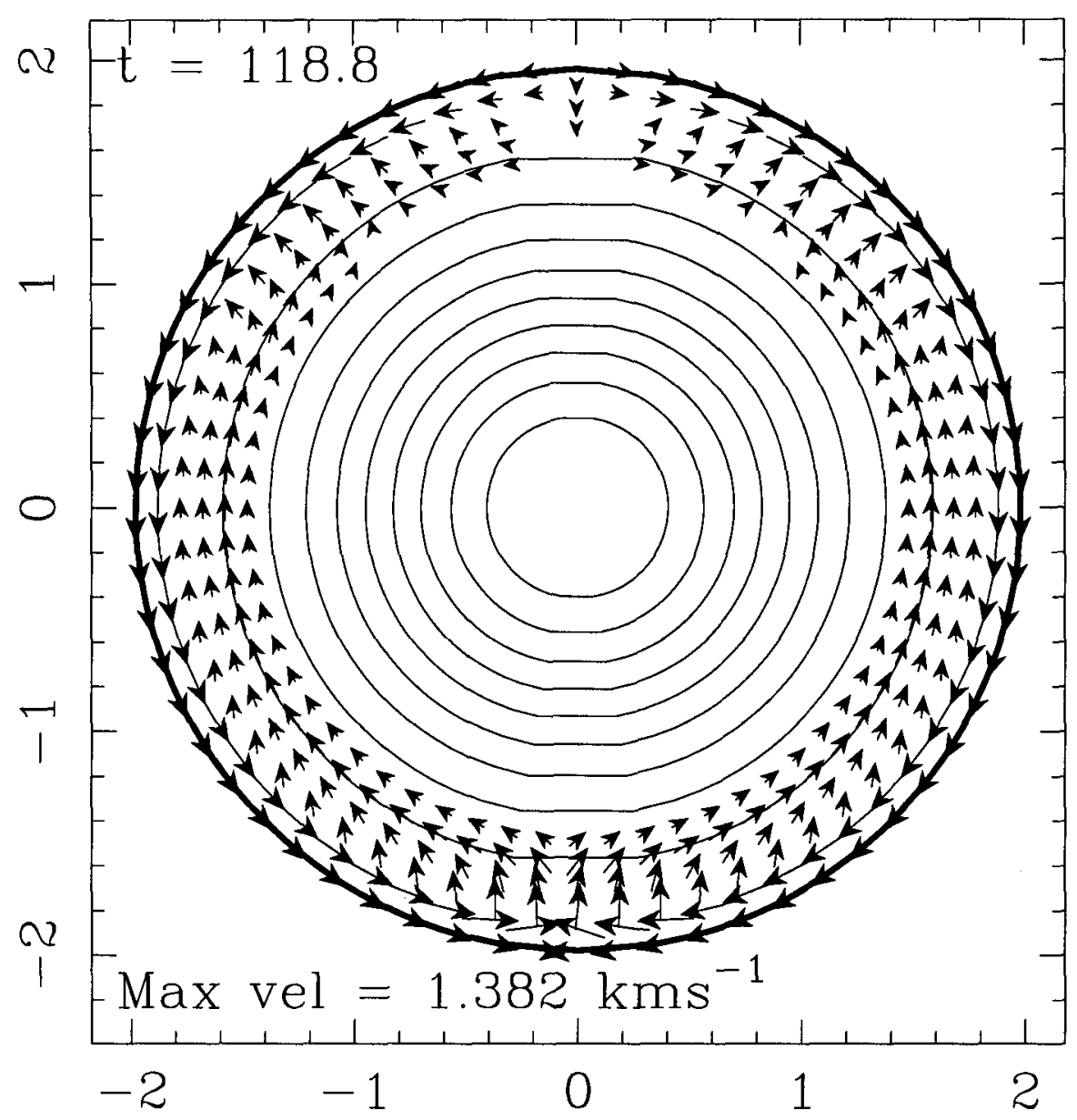

Figure 1. The closed circulation pattern reached in an irradiated star. The irradiation was modelled as an applied force in the uppermost layer of the star. The length of the arrows is proportional to the log of their velocities. 
Drechsel, H., Haas, S., Lorenz, R., \& Gayler, S. 1995, A\&A, 294, 723

Hameury, J.-M. 1996, A\&A, 305, 468

Hessman, F. V., Robinson, E. L., Nather, R. E., \& Zhang, E.-H. 1984, ApJ, 286, 747

Martin, T. J., \& Davey, S. C. 1995, MNRAS, 275, 31

Kippenhahn, R., \& Thomas, H. C. 1979, A\&A, 75, 281

Kırbıyık, H. 1982, MNRAS, 200, 907

Kırbıyı, H., \& Smith, R. C. 1976, MNRAS, 176, 103

Orosz, J. A., \& Bailyn, C. D. 1997, ApJ, 477, 876

Pedlosky, J. 1987, Geophysical Fluid Dynamics (New York: Springer-Verlag)

Phillips, S. N., Shahbaz, T., \& Podsiadlowski, Ph. 1999, MNRAS, 304, 839

Phillips, S. N. \& Podsiadlowski, P. 2002, MNRAS, submitted (astro-ph/0109304)

Podsiadlowski, Ph. 1991, Nature, 350, 136

Ritter, H., Zhang, Z.-Y., \& Kolb, U. 2000, A\&A, 360, 959

Ruderman, M., Shaham, J., \& Tavani, M. 1989, ApJ, 336507

Shahbaz, T., Groot, P., Phillips, S. N., Casares, J., Charles, P. A., \& van Paradijs, J. 2000, MNRAS, 314, 747

Wilson, R. E. 1973a, ApJ, 181, L75

Wilson, R. E. 1973b, ApJ, 184, L99 